\title{
Many-body blockade of resonant tunneling of two-dimensional electrons
}

\author{
J. G. S. Lok, A. K. Geim, and J. C. Maan \\ High Field Magnet Laboratory, Research Institute for Materials, University of Nijmegen, 6525 ED Nijmegen, The Netherlands \\ L. Eaves, A. Nogaret, P. C. Main, and M. Henini \\ Department of Physics, University of Nottingham, Nottingham NG7 2RD, England
}

(Received 13 March 1997)

\begin{abstract}
In high magnetic fields tunneling of two-dimensional electrons requires an extra energy to overcome an effective barrier due to electron-electron interaction. This barrier is clearly visible as a shift of the tunnel resonance to higher biases. The filling factor $\nu=1$ marks a surprisingly rapid transition between low-field (quadratically developing shift) and high-field (saturation) regimes. At low temperatures, the many-body shift decays linearly with increasing temperature, which can be attributed to coupling of a tunneling electron to phononlike excitations in the correlated electron system. [S0163-1829(97)04327-7]
\end{abstract}

Since the pioneering work of Chang, Esaki, and $\mathrm{Tsu}^{1} \mathrm{a}$ great deal of attention has been paid to studying physics of resonant tunneling and the single-particle approach is found to be generally adequate to describe this long familiar system. However, it has recently become clear that many-body phenomena can also play a substantial role in resonant tunneling and even lead to qualitative changes in its behavior. Such changes have been observed in two specific situations: as a Fermi-edge singularity for the tunneling of twodimensional (2D) electrons through a strongly localized state of a single impurity ${ }^{2}$ and as a plasmon replica in asymmetric resonant tunneling devices (RTD's) in the regime of change accumulation. $^{3}$

In this paper, we show that electron-electron interaction is also responsible for another qualitative feature which can readily be seen in the behavior of conventional RTD's with a two-dimensional electron gas (2DEG) at the emitter interface. The interaction leads to a clearly noticeable shift of the tunnel resonance to higher biases when a strong magnetic field is applied perpendicular to the 2DEG. Such a shift indicates the emergence of an extra barrier that restrains electron tunneling. We explain this shift in terms of the so-called Coulomb (quasi)gap, which has recently been observed for equilibrium tunneling between two 2D-electron systems $\mathrm{s}^{4,5}$ and, subsequently, attracted significant theoretical interest. ${ }^{6-8}$ The observation of the quasigap as a first-order effect in resonant tunneling that survives up to temperatures of several tens of Kelvin is only one of the points of this paper. ${ }^{9}$ Further, the process which controls the tunnel current in our RTD's is tunneling from the emitter 2DEG into the empty quantum well. This essentially distinguishes our system from double-layer devices ${ }^{4,5}$ giving further information about the nature of the Coulomb quasigap. First, the evidence for the many-body blockade in the system with a single 2DEG proves that the intralayer rather than interlayer interaction dominates this effect. ${ }^{8}$ Second, a free supply of electrons into the emitter 2DEG from the parallel planar contact layer effectively removes the strongly oscillating in-plane 2DEG magnetoresistance which has hampered the accuracy of measurements in the double 2DEG systems in low magnetic fields. In our system, we have been able to accurately mea- sure magnetic-field and temperature dependences of the quasigap which both appear to be remarkable. In high magnetic fields, the many-body shift exhibits saturation to a value of about $\Delta(T=0) \approx e^{2} / r$ where $r$ is the average electron spacing in the $2 \mathrm{DEG}$, in agreement with the previous work on double 2DEG systems. In low fields, we have found a quadratic dependence on $B$ which cannot be explained by current theories. The transition point between the two regimes is clearly marked by the filling factor $\nu=1$ and cannot be connected to any other meaningful filling factor, e.g., $\nu=2$ or $\frac{2}{3}$. This is an unambiguous indication that the quasigap involves spin correlations. Third, the observed temperature dependence of the interaction shift is well described by the unusual exponential dependence $\Delta(0) \exp \left[-\alpha k_{B} T / \Delta(0)\right](\alpha$ $\approx 1)$ within the whole temperature range. At low temperatures, this yields the linear dependence $\Delta(T) \approx \Delta(0)-k_{B} T$ which, we speculate, indicates contribution to the tunnel process of thermally excited phononlike plasmons inside the correlated electron system.

We have studied magnetotunneling of 2D electrons using (AlGa)As double-barrier RTD's. A schematic band diagram of our devices under bias is shown in the inset of Fig. 1. Due to the presence of an undoped spacer layer between the (AlGa)As emitter barrier and the $n$-doped contact region, a 2DEG is formed in the accumulation layer at the emitter interface. Tunneling occurs when the 2DEG comes in resonance with a $2 \mathrm{D}$ subband in the quantum well giving rise to a sharp peak on the $I-V$ curve (Fig. 2). Our devices were grown by molecular-beam epitaxy and have the peak-tovalley ratio for the main resonance of about 20 at $4 \mathrm{~K}$, indicating their high quality. A large number of devices with various layer compositions (different thicknesses of tunnel barriers, the quantum well and spacer layers, and various doping profiles) have been studied. Here, we focus on data for two of our structures which fairly represent the range of possible behavior in this system. For the first sample, the active region consists of an 11- $\mathrm{nm} \mathrm{Al}_{0.4} \mathrm{Ga}_{0.6} \mathrm{As}$ barrier, a 5.8-nm GaAs quantum well followed by another 8.3-nm $\mathrm{Al}_{0.4} \mathrm{Ga}_{0.6} \mathrm{As}$ barrier with 3.4-nm space layers. The second device has a 9.0-nm GaAs quantum well sandwiched between two $5.7-\mathrm{nm} \mathrm{Al}_{0.3} \mathrm{Ga}_{0.7} \mathrm{As}$ barrier followed by $20-\mathrm{nm}$ 


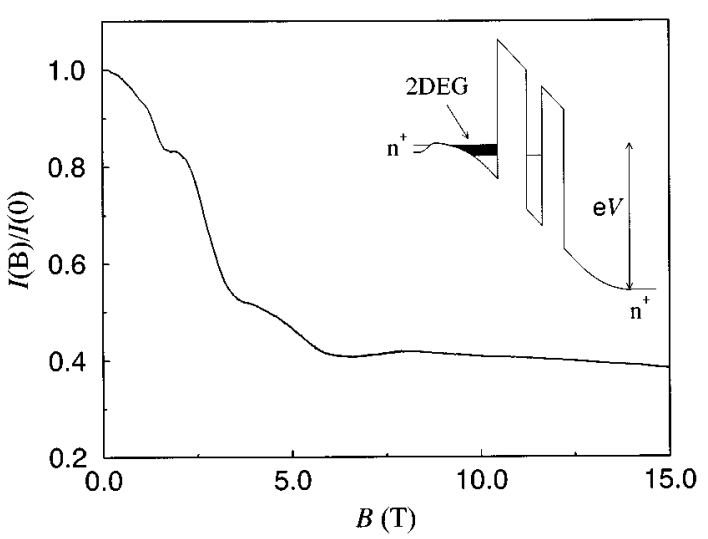

FIG. 1. Suppression of the tunnel current by a strong magnetic field parallel to the current at biases near the onset of the resonance ( $\nu=1$ at $B \approx 6 \mathrm{~T}$ ). Inset: schematic diagram of the conduction-band profile of our devices under bias. Electron tunneling occurs from the emitter 2DEG through the 2D subband in the quantum well.

spacer layers. The doping starts from a low value (2 $\times 10^{16} \mathrm{~cm}^{-3}$ ) over thicknesses of 100 and $300 \mathrm{~nm}$ close to the spacer layers, for the two devices, respectively. We discuss the situation when the current is controlled by tunneling through the emitter barrier and serial resistances due to other parts of the structure can be neglected. For further details we refer to Refs. 2 and 10.

When measuring the tunnel current at a fixed bias below the peak of the main resonance, the current always first rapidly decreases with increasing magnetic field and, then, this is followed by a saturation or slower decrease in fields above $\nu=1$. Figure 1 shows that this is a primary effect with the current being suppressed by a factor of 2.5 (Fig. 1) or more. ${ }^{11}$ Full $I-V$ characteristics measured for different values of the magnetic field reveal that the suppression of tunneling is simply due to the shift of the whole body of the resonance to higher biases (see Fig. 2). The inset shows that the shift is really "rigid," covering the whole range of the exponential increase in the tunnel current down to the regime of impurity-related tunneling. ${ }^{2,11}$ From the phenomenological point of view the magnetic field acts as if the energy of the 2D subband in the quantum well (the Fermi level of the 2DEG) is moved upwards (downwards) with increasing

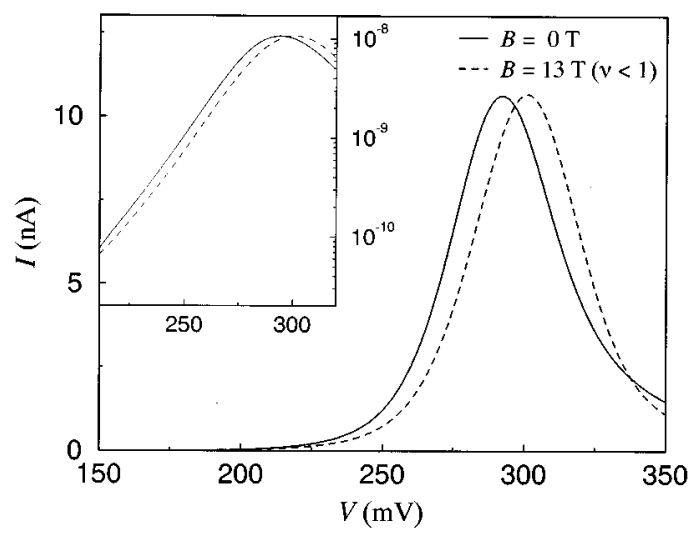

FIG. 2. Parallel shift of the tunnel resonance to higher biases when a quantizing magnetic field is applied to the 2DEG. Inset: the same resonance in the logarithmic scale. magnetic field. The shift of the main resonance may sometimes be accompanied by changes in its amplitude; however, if the resonance is normalized to the peak value, the rigid shift of the front part of the resonance is always evident. The part beyond the resonance usually experiences some changes as the resonance becomes more symmetric in high magnetic fields, in agreement with theory. ${ }^{12}$

Another important experimental fact which elucidates the nature of the observed shift is that the value of the magnetic field where the saturation occurs corresponds to filling factor 1 in the emitter 2DEG (filling factor is found from the period of the magneto-oscillations). This indicates that the shift is related to quantization in the 2DEG. Furthermore, rotating the field direction relative to the plane of the emitter 2DEG confirms that the shift is $2 \mathrm{D}$ in nature. The magnitude of the shift is found to be several $\mathrm{mV}$ depending on the layer composition and 2D electron concentration $n$ in different devices. Converting the voltage shift into the energy, ${ }^{2,10}$ we find that at low temperatures the additional energy $\Delta$ required for resonant tunneling in high fields varies between 2 and $5 \mathrm{meV}$ for different devices with $n$ between 0.7 and 3.0 $\times 10^{11} \mathrm{~cm}^{-2}$. Note that $\Delta$ does not correspond to any other obvious energy scale in the problem. The energy $\Delta$ increases with increasing $n$ and, within the data scatter for different devices, can be described by the dependence $\Delta(0)=\beta e^{2} / r$ $\propto n^{1 / 2}(\beta \approx 0.6-0.8) .^{4-8}$

As mentioned above, the observed shift of the tunnel resonance to higher bias is not expected in a simple singleparticle theory of resonant tunneling. ${ }^{13}$ In fact, the onset of the resonance is due to $2 \mathrm{D}$ electrons at the Fermi level which tunnel with energy conservation but without momentum conservation due to the presence of impurity and interface scattering. On the other hand, the 2D Fermi level is pinned to the Fermi level in the doped contact region because the corresponding series resistance of the spacer layers is negligibly small. Since the zero-point energy shift $h \omega_{0} / 2$ (lowest Landau level) is the same for all parts of the structure, the singleparticle theory of magnetotunneling predicts that the onset of the tunnel resonance is independent of the value of the perpendicular magnetic field. We note that we considered a number of other reasons which could speculatively lead to a shift of the resonance. Among those are magnetoresistance of the spacer regions or the emitter $2 \mathrm{DEG}$, change in the shape of the emitter confinement, tunneling with momentum conservation, nonparabolicity of the energy spectrum in the quantum well, and misalignment of the magnetic field with respect to the 2DEG. All such speculation are in qualitative disagreement with the discussed behavior or have been ruled out by additional experiments (e.g., rotation in the magnetic field, changing its polarity, and capacitance measurements).

A straightforward explanation for the observed shift is given by employing the concept of the Coulomb quasigap observed in recent experiments on equilibrium tunneling of $2 \mathrm{D}$ electrons. ${ }^{4,5}$ The many-body gap can be interpreted as being due to local excitations left in a 2D system by rapid extraction of a tunneling electron. In high magnetic fields 2D electrons become strongly correlated as the magnetic field quenches their kinetic energy. Then, extraction of an electron out of the correlated system leaves local plasmon excitations. Hence, an additional energy (or bias) has to be supplied to the system to provide the energy conservation in the whole 


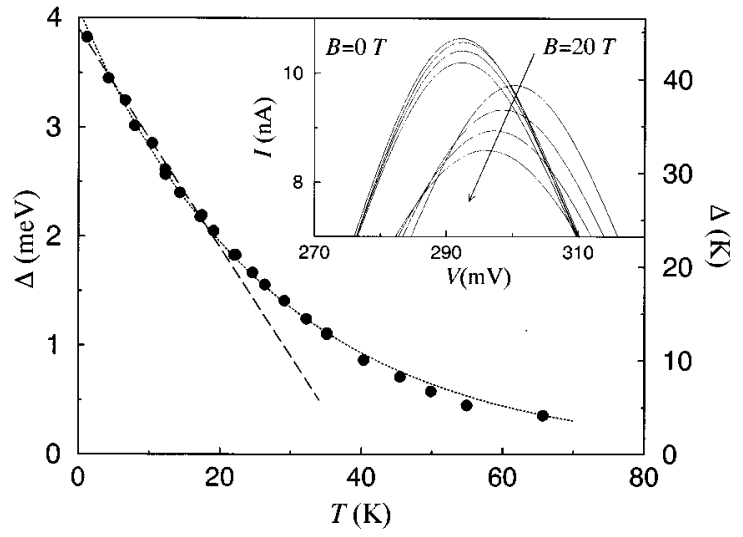

FIG. 3. Temperature dependence of the energy required to overcome the additional barrier caused by the magnetic field. Experimental data are shown by dots; the solid line is the best fit by the function $\Delta(0) \exp \left[-\alpha k_{B} T / \Delta(0)\right]$ with $a \approx 1$; the dashed line is the linear suppression $\Delta(T)=\Delta(0)-k_{B} T$. The inset shows in more detail the evolution of the resonance position with increasing temperature in the fields of 0 and $20 \mathrm{~T}\left(\nu=\frac{1}{2}\right)$.

tunnel process which now includes the plasmon emission. The value of the "gap" corresponds to the interaction energy $e^{2} / r$ and the gap appears in high magnetic fields and at low temperatures when the 2D system is strongly correlated. Applying this concept to our experimental system explains the observed shift, its absolute value, and the concentration dependence. $^{4-8}$

We have used the new experimental situation (single 2DEG; vertical supply of electrons) to study the behavior of the many-body blockade at high temperatures and in low magnetic fields. The temperature dependence is plotted in Fig. 3. As expected, ${ }^{5}$ the thermal energy $k_{B} T$ required to suppress the blockade is of the same order of magnitude as the gap itself, $\Delta(0) / k_{B} \approx 40 \mathrm{~K}$ (Fig. 3). The resonance becomes slightly smaller (and broader) with increasing temperature up to $70 \mathrm{~K}$ but its position in zero magnetic field is temperature independent (see inset in Fig. 3). On the other hand, in high magnetic fields the resonance shifts to lower biases with increasing temperature, gradually approaching its zero-field position. This unambiguously indicates that all the additional energy is related to the magnetically quantized state of the 2D system and the blockade of tunneling is absent in zero field. Further, the temperature dependence of the shift of the resonance shown in Fig. 3 can be well described by the exponential function $\Delta(0) \exp \left[-k_{B} T / \Delta(0)\right]$, which is drastically different from the Arrhenius dependence exp $\left[-\Delta(0) / k_{B} T\right]$ or the Fermi function. In the absence of a theory for such behavior, we note that at low temperatures $\left[T \ll \Delta(0) / k_{B}\right]$ the experimental dependence is also well described by the linear function $\Delta(T)=\Delta(0)-k_{B} T$ (dashed line in Fig. 3). We speculate that this dependence suggests phononlike excitations in the strongly correlated electron system. Such phonons (analogous to acoustic phonons but in an electron glass) have a characteristic energy $k_{B} T$ and can couple with tunneling electrons, thus supplying the extra energy of the order of $k_{B} T$ necessary to overcome the blockade. At higher temperatures $\left(k_{B} T \approx \Delta\right)$, the correlated system melts and the gap completely disappears.

Figure 4 shows the magnetic-field dependence of the

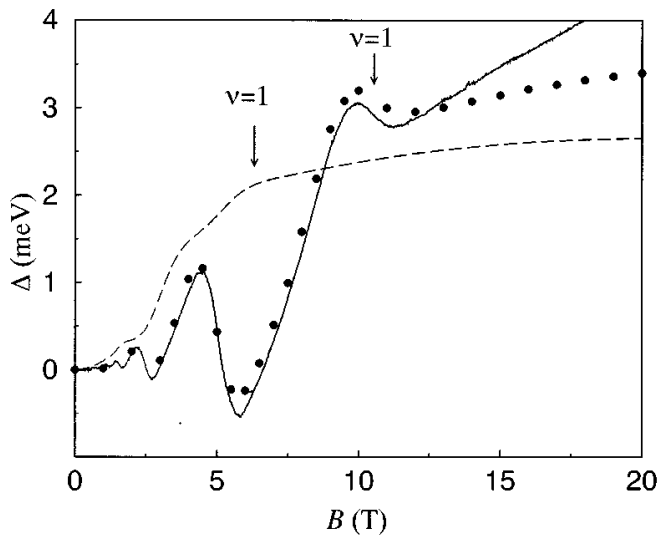

FIG. 4. Development of the interaction barrier with increasing magnetic field at $4.2 \mathrm{~K}$ for two samples with different electron concentrations. Curves are in situ measurements at a fixed value of the tunnel current. The dotted curve takes into account changes in the amplitude of the resonance for one of the samples.

quasigap over the field interval from 0 to $20 \mathrm{~T}$ for the two samples discussed. The $2 \mathrm{DEG}$ concentrations differ by a factor of about 2. The solid and dashed lines are obtained directly by fixing the tunnel current and sweeping the magnetic field. This procedure accurately measures the shift of the tunnel resonance if its form does not change. This is the case of the device depicted by the dashed curve in Fig. 4. For the solid curve, the amplitude of the resonance slightly decreases in fields above $12 \mathrm{~T}$ and this leads to the artifact that the solid curve goes up. The real shift is obtained by normalizing the resonance amplitudes in different fields (dotted curve). One can see that with increasing field, the gap develops nonmonotonically and can exhibit strong magneto-oscillations. The exact amplitude and phase of these oscillations are beyond accuracy of the present experiment because it is possible that simultaneously there is an oscillating contribution to the measured signal due to a self-consistent adjustment of the accumulation layer. ${ }^{13}$ We note that at low temperatures $(\approx 0.4 \mathrm{~K})$ a small but clear increase in the value of the gap is observed at $\nu=\frac{2}{3}$ and $\frac{1}{3}$.

When the amplitude of the oscillations in the value of the gap is small, two field regimes can be clearly distinguished. In low fields, the gap is described by a quadratic dependence, while in high fields it rapidly saturates. The quadratic growth of the quasigap is predicted in Ref. 7 but the rate of this increase is one order of magnitude larger than that measured in our experiment. We note that the theory ${ }^{7}$ considers the clean limit in the 2DEG while in our structures impurity scattering is perhaps important at the onset of the resonance. In high magnetic fields $(\nu<1)$, there is a smaller increase in the gap value which can be described as a square-root dependence offset from the origin at zero field. Such a weakfield dependence is expected because the magnetic length $l_{H}$ still does not reach the classical limit for the interacting system $\left(l_{H} \ll r\right)$. Note that the transition between the two field regimes is remarkably sharp and for all samples is determined by the filling factor $\nu=1$ and not by the absolute value of the magnetic field. The filling factor $\nu=1$ is marked by arrows in Fig. 4 and it is clear that the transition between the two field regimes occurs at the spin-splitted state and not at $\nu=2$. This indicates that the observed "gap" is 
not only related to the Coulomb interaction between electrons but that also their spin correlations are involved.

In conclusion, many-body interaction causes considerable and easily observable changes in $I-V$ characteristics of conventional resonant tunneling devices. This phenomena survives even at elevated temperatures of several $10 \mathrm{~K}$. The temperature dependence cannot be explained by present theories and indicates coupling of the initial state of the tunnel electron with phononlike excitations in the correlated electron system. The point of the crossover between the low- and high-field regimes indicates that spin correlations are important for the description of the quasigap. We observe a quadratic evolution of the gap in low fields but its amplitude is considerably smaller than predicted.

This work is part of a research program of the Stichting voor Fundamenteel Onderzoek der Materie (FOM) financially supported by NWO (The Netherlands) and was also supported by EPSRC (UK).
${ }^{1}$ L. L. Chang, L. Esaki, and R. Tsu, Appl. Phys. Lett. 24, 593 (1974).

${ }^{2}$ A. K. Geim, P. C. Main, N. La Scale, L. Eaves, T. J. Foster, PH. Beton, J. W. Sakai, F. W. Sheard, M. Henini, G. Hill, and M. A. Pate, Phys. Rev. Lett. 72, 2061 (1994).

${ }^{3}$ C. Zhang, M. L. F. Lerch, A. D. Martin, P. E. Simmonds, and L. Eaves, Phys. Rev. Lett. 72, 3397 (1994).

${ }^{4}$ R. C. Ashoori, J. A. Lebens, N. P. Bigelow, and R. H. Silsbee, Phys. Rev. Lett. 64, 681 (1990).

${ }^{5}$ J. P. Eisenstein, L. N. Pfeifer, and K. W. West, Phys. Rev. Lett. 69, 3804 (1992); K. M. Brown, N. Turner, J. T. Nicholls, E. H. Linfield, M. Pepper, D. A. Ritchie, and G. A. C. Jones, Phys. Rev. B 50, 15465 (1994).

${ }^{6}$ S. R. E. Young and A. H. MacDonald, Phys. Rev. Lett. 70, 4110 (1993); S. He, P. M. Platzman, and B. I. Halperin, ibid. 71, 777 (1993); P. Johansson and J. M. Kinaret, ibid. 71, 1435 (1993); R. Haussman, H. Mori, and A. H. MacDonald, ibid. 76, 979 (1996).

${ }^{7}$ I. L. Aleiner, H. U. Baranger, and L. I. Glazman, Phys. Rev. Lett. 74, 3435 (1995).

${ }^{8}$ C. M. Varma, A. I. Larkin, and E. Abrahams, Phys. Rev. B 49, 13999 (1994); G. E. W. Bauer, Surf. Sci. 305, 358 (1994).
${ }^{9}$ Note that the Coulomb quasigap is to be distinguished from the more common single-electron Coulomb blockade in mesoscopic systems where charging of a small capacitor plays a role.

${ }^{10}$ J. W. Sakai et al., Phys. Rev. B 48, 5664 (1993); M. L. Leadbeater et al., J. Phys. Condens. Matter 1, 10605 (1989).

${ }^{11}$ Suppression of tunnel current in high fields has been observed in several experiments but there the attention was concentrated on Shubnikov-de Haas-like oscillations and the changing background was not discussed. M. L. Leadbeater, Ph.D. thesis, University of Nottingham (1990); J. W. Sakai, T. M. Fromhold, P. H. Beton, M. Henini, L. Eaves, P. C. Main, F. W. Sheard, and G. Hill, Physica B 184, 241 (1993); L. D. Macks, S. A. Brown, R. G. Clark, R. P. Starrett, M. A. Reed, M. R. Deshpande, C. J. L. Fernando, and W. R. Frensley, Phys. Rev. B 54, 4857 (1996). Note that the parallel shift is sometimes obscured by additional changes in the form of the tunnel resonance.

${ }^{12}$ J. Leo and A. H. MacDonald, Phys. Rev. B 43, 9763 (1991).

${ }^{13}$ M. Kaplit and J. N. Zimel, Phys. Rev. Lett. 21, 212 (1968); K. S. Chan, F. W. Sheard, G. A. Toombs, and L. Eaves, Superlattices Microstruct. 9, 23 (1990). 